Article

\title{
Nanostructure Design and Catalytic Performance of Mo/ZnAl-LDH in Cationic Orchid X-BL Removal
}

\author{
Yin $\mathrm{Xu}$ *, Tingjiao Liu, Yang Li, Yun Liu and Fei Ge \\ Department of Environment, College of Environment and Resources, Xiangtan University, \\ Xiangtan 411105, China; xtu_ltj2016@126.com (T.L.); xtu_ly2012@163.com (Y.L.); liuyunscut@163.com (Y.L.); \\ gefei@xtu.edu.cn (F.G.) \\ * Correspondence: xuyin@xtu.edu.cn; Tel.: +86-731-5829-2231
}

Received: 3 November 2018; Accepted: 25 November 2018; Published: 27 November 2018

\begin{abstract}
The nanostructure of ZnAl-layered double hydroxide (ZnAl-LDH) was designed to promote the catalytic performance of Mo-based ZnAl-LDH (Mo/ZnAl-LDH) catalysts, in a catalytic wet air oxidation (CWAO) process, under room temperature and pressure, in degradation of dye wastewater. Four most commonly used preparation methods, traditional precipitation (TP), hydrothermal synthesis (HS), sol-gel (SG), and urea co-precipitation (UC) were employed to prepare the $\mathrm{ZnAl}-\mathrm{LDH}$. The resulting Mo/ZnAl-LDH samples were contrasted through surface area, crystal structure, chemical state, and morphology. The degradation of cationic orchid X-BL, under room temperature and pressure, was developed to determine the catalytic activity of these Mo/ZnAl-LDH samples. The results showed that the nanostructure of $\mathrm{ZnAl}-\mathrm{LDH}$, prepared by HS, enhanced the adhesion of the catalytic active component, thus Mo/ZnAl-LDH had the highest catalytic activity of $84.2 \%$ color removal efficiency and $73.9 \%$ total organic carbon removal efficiency. Specific Mo species, such as $\mathrm{Na}_{2} \mathrm{Mo}_{2} \mathrm{O}_{7}$, Mo dispersion, and $\mathrm{O}^{2-}$ ions were proved to be related with catalytic performance. These findings preliminarily clarified that LDHs preparation methods make a difference in the performance of Mo/LDHs.
\end{abstract}

Keywords: preparation method; Mo/ZnAl-LDH catalyst; organic wastewater; room conditions; wet catalytic oxidation

\section{Introduction}

Catalytic wet air oxidation (CWAO) is an effective method for organic wastewater treatment in the 21st century [1,2], which allows the use of less severe reaction conditions. In addition, it is suited to the decomposition of refractory pollutants, even, thereby, reducing the capital and operating costs [3]. The choice of the catalyst in the CWAO process is significant and, thus, seeking an effective, robust, and low-cost catalyst, has been a research hotspot [4-6]. Heterogeneous catalysts containing small amounts of molybdenum oxides, and clay-immobilized molybdenum, have attracted considerable attention because of their wide structural variety and specific properties. Our previous studies show that a Mo-Zn-Al-O catalyst has an excellent performance on the degradation of dye wastewater, under room temperature and pressure [7,8]. To promote the catalytic activity of Mo-Zn-Al-O catalyst, attention has been focused on the nanostructure design.

Generally, the catalytic performance of catalyst is determinedly influenced by their structure and morphology. During the preparation process, many factors have an impact on the final catalyst properties [9-14]. Specially, the nanostructure of the carrier, affect the metal dosage because of the interactions between the carrier and the metal precursor [15-17]. Layer double hydroxides (LDHs) have found an increasingly wide utilization in the catalyst carrier. Their main structure features are highly-related to their preparation method [18]. The most common synthesis method is the traditional 
precipitation (TP), at a constant $\mathrm{pH}$. The urea co-precipitation (UC), sol-gel (SG), and hydrothermal synthesis (HS) create an alkaline environment and well-crystallized LDHs. Another popular method is the reconstruction from oxide generated by calcining LDHs, taking advantage of the "memory effect" also allows the intercalation of any desired anion, such as biomedical or active metal anions. Although the way to prepare LDHs was reported to have a significant effect on the final properties [19], the details of the relationship between the nanostructure of LDHs carrier, and the active metal precursor, are not fully understood.

It is reasonable to believe that the control of interaction between the active Mo component and LDHs carrier is a new direction for the newly-developed CWAO catalyst. Four typical methods were adopted for the ZnAl-LDH preparation, in the present study. The as-prepared samples were characterized by $\mathrm{N}_{2}$ adsorption-desorption, X-ray diffraction, $\mathrm{X}$-ray photoelectron spectrum, and transmission electron microscopy. In brief, the cationic orchid X-BL was harmful to human health, under certain conditions, which was representative of dye wastewater [20]. Thus, their catalytic efficiency on the cationic orchid X-BL degradation was investigated, in detail. We optimized the formation to obtain a Mo/ZnAl-LDH catalyst with a high catalytic performance, on the basis of these results.

\section{Materials and Methods}

\subsection{Materials}

Cationic orchid X-BL was purchased from the Shanghai Luojing Dyeing Chemical Co., Ltd. (China). The chemicals used for this project, including $\mathrm{Zn}\left(\mathrm{NO}_{3}\right)_{2} \cdot 6 \mathrm{H}_{2} \mathrm{O}(99 \%), \mathrm{Al}\left(\mathrm{NO}_{3}\right)_{3} \cdot 9 \mathrm{H}_{2} \mathrm{O}(99 \%)$, $\mathrm{NaOH}(99 \%), \mathrm{C}_{6} \mathrm{H}_{8} \mathrm{O}_{7} \cdot \mathrm{H}_{2} \mathrm{O}(99.5 \%), \mathrm{CH}_{4} \mathrm{~N}_{2} \mathrm{O}(99 \%)$, and $\left(\mathrm{NH}_{4}\right)_{6} \mathrm{Mo}_{7} \mathrm{O}_{24} \cdot 4 \mathrm{H}_{2} \mathrm{O}(99 \%)$, were purchased from the Sinopharm Chemical Reagent Co. Ltd. (Shanghai, China) They were used, as received, without further purification. Deionized water was used for all preparations.

\subsection{Preparation of $\mathrm{ZnAl-LDH}$ Carriers}

\subsubsection{The TP Process}

Sodium hydroxide solution (20\%) was added drop-wise to a vigorously stirred, mixed solution, containing the $\mathrm{Zn} / \mathrm{Al}$ mixed solution (1:1), at constant $\mathrm{pH}$ of 9.5-10. After the resulting slurry was aged at $80^{\circ} \mathrm{C}$, for $18 \mathrm{~h}$, the wet cake was thoroughly filtered and rinsed with deionized water. Finally, the sample was dried at $70{ }^{\circ} \mathrm{C}$, for $12 \mathrm{~h}$, and gently ground into the LDH-TP.

\subsubsection{The HS Process}

Different from the TP, the sodium hydroxide solution and the $\mathrm{Zn} / \mathrm{Al}$ mixed solution (1:1) were blended rapidly, in the process of HS. Afterwards, the formed slurry was transferred into stainless Teflon-lined $100 \mathrm{~mL}$ capacity autoclaves, then sealed and maintained at $120^{\circ} \mathrm{C}$, for $24 \mathrm{~h}$, in an oven. After the hydrothermal treatment, the autoclaves were cooled down to the room temperature, filtered, washed thoroughly with deionized water, dried, and ground gently into the LDH-HS.

\subsubsection{The SG Process}

Citric acid (2.5 mol/L) used as a binder was added into the $\mathrm{Zn} / \mathrm{Al}$ mixed solution (1:1), through a peristaltic pump. Subsequently, an opaque faint yellow solution was finally observed, as the obtained mixed solution was stirred in $80^{\circ} \mathrm{C}$ water bath, for $10 \mathrm{~h}$, to evaporate the solvent. Moreover, the final sol was dried at $120^{\circ} \mathrm{C}$ for $48 \mathrm{~h}$, and ground gently into the LDH-SG. 


\subsubsection{The UC Process}

A solution of the $\mathrm{Zn} / \mathrm{Al}$ mixed solution (1:1) was mixed with a urea solution $(7.5 \mathrm{~mol} / \mathrm{L})$ in a $1000 \mathrm{~mL}$ round bottom flask, put into an oil bath at $120^{\circ} \mathrm{C}$; stirred and reflowed for $10 \mathrm{~h}$. Then, the final slurry was filtered, washed, dried, and ground gently into the LDH-UC.

\subsection{Mo/ZnAl-LDH Catalysts Preparation}

The ZnAl-LDH support was impregnated in a $20 \mathrm{~mL}$ aqueous solution, containing $0.28 \mathrm{~mol} / \mathrm{L}$ ammonium heptamolybdate. The $\mathrm{pH}$ of the mixture was maintained at 8 and the solution was maintained at room temperature for $12 \mathrm{~h}$. After that, the resulting product was dried at $80{ }^{\circ} \mathrm{C}$ for $10 \mathrm{~h}$ and calcined at $400{ }^{\circ} \mathrm{C}$ for $1 \mathrm{~h}$. The resulting solid prepared using the four kinds of preparation methods of support, were marked as Mo-TP, Mo-HS, Mo-SG and Mo-UC, respectively.

\subsection{Characterization}

The $\mathrm{N}_{2}$ adsorption-desorption on the surface of the Mo/ZnAl-LDH catalysts was measured on the Quantachrome NOVA-2000E apparatus (Quantachrome, FL, USA). By analysis of the adsorption-desorption isotherm of nitrogen, through the Brunauer-Emmett-Teller (BET) approach, the specific surface area of the samples was obtained. The pore size and volume distribution were calculated by the Brunauer-Joyner-Halenda (BJH) method. Before obtaining the adsorption-desorption isotherms, all catalysts were outgassed at a temperature of $150{ }^{\circ} \mathrm{C}$.

X-ray diffraction (XRD) patterns of the catalyst was obtained on a Philips Panalytical X'Pert PRO X-ray diffractometer (PANalytical, Almelo, The Netherlands), using $\mathrm{Cu} \mathrm{K} \alpha$ radiation, at a scan rate of $0.022 \theta \mathrm{s}^{-1}$ in the $2 \theta$ range of $5^{\circ}-80^{\circ}$ to determine the phase structures of the obtained samples. The applied current and accelerating voltage were $30 \mathrm{~mA}$ and $40 \mathrm{kV}$, respectively. The Joint Committee of Powder Diffraction Standard (JCPDS) cards were used to manually analyze the diffraction patterns.

Zeta-potentials were measured using a zeta-potential analyzer (Brookhaven ZetaPALS, New York, NY, USA) and each value was reported as an average of five measurements.

X-ray photoelectron spectroscopy (XPS) was recorded on a Thermo Fisher Scientific ESCALAB 250 X-ray spectrometer (Waltham, MA, USA), fitted with a monochromatic Al excitation source (photon energy was at $1486.6 \mathrm{eV}$ ), operating at a transmitter current of $10 \mathrm{~mA}$ and a voltage of $15 \mathrm{kV}$. All spectra were calibrated to the $\mathrm{C} 1 \mathrm{~s}$ peak (binding energy $=284.6 \mathrm{eV}$ ) for the adventitious carbon, and all the datum of XPS were analyzed, using the Casa 2273 XPS software (Casa XPS 2.3.15, Quayside bookshop, Teignmouth, UK).

The morphology and particle size of the catalysts were characterized by Transmission electron microscopy (JEM-2100, JEOL, Tokyo, Japan), operated with an accelerating voltage of $200 \mathrm{kV}$, and magnification ranges of 0.5-1.5 million times. All the sample specimens for TEM studies were ultrasonically dispersed in ethanol, and one or two drops of the upper layer solution was pipetted out on a micro-mesh cupper grid. Before the TEM analysis, the sample specimens were vacuum dried in the microscope column, for 7-8 min.

\subsection{Catalytic Activity Evaluation}

In the CWAO process, cationic orchid X-BL was the representative of the organic wastewater. Figure 1 shows the structure of the cationic orchid X-BL. The degradation of the cationic orchid X-BL by the Mo/ZnAl-LDH catalyst was evaluated, using a simple beaker device, under room temperature and pressure. A sample of $1 \mathrm{~g} / \mathrm{L} \mathrm{Mo} / \mathrm{ZnAl}-\mathrm{LDH}$ catalyst was used to degrade $100 \mathrm{mg} / \mathrm{L}$ cationic orchid X-BL, with an air flux of $3.5 \mathrm{~L} / \mathrm{min}$. The $\mathrm{pH}$ of the simulated industry wastewater was not adjusted. During the CWAO process, the decomposition rate of cationic orchid X-BL was estimated on the basis of the absorbency, by UV-vis spectrophotometer (Shimadzu UV 2550, Kyoto, Japan) and Total Organic Carbon (TOC) analyzer (Shimadzu CPHCN200, Kyoto, Japan), to evaluate the mineralization of the cationic orchid X-BL, after reaction. 


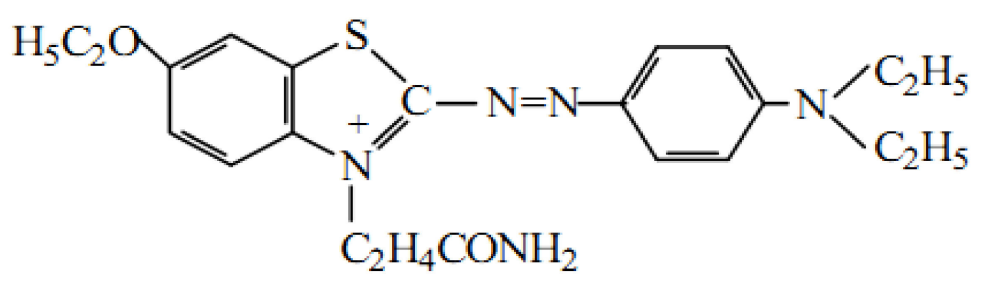

Figure 1. The structure of the cationic orchid X-BL.

\section{Results and Discussion}

\subsection{Crystal Structure of Mo/ZnAl-LDH Catalysts}

The crystal phases of all as-prepared Mo/ZnAl-LDH catalyst are shown in Figure 2. All catalyst exhibited the diffraction lines arising from the $\mathrm{Na}_{2} \mathrm{MoO}_{4}$ phase (JCPDS 12-0773), located at $2 \theta=16.95^{\circ}$, $27.76^{\circ}, 35.66^{\circ}, 48.98^{\circ}, 52.18^{\circ}$, and $57.27^{\circ}$. Mo-SG and Mo-UC have extremely sharp and narrow diffraction spectra and exhibit high characteristic peaks of $\mathrm{Na}_{2} \mathrm{MoO}_{4}$, which indicates that Mo-SG and Mo-UC contain a single substance. However, Mo-TP and Mo-HS have extremely short and wide diffraction spectra and exhibit low characteristic peaks of $\mathrm{Na}_{2} \mathrm{Mo}_{2} \mathrm{O}_{7}$ (JCPDS 22-0906) and $\mathrm{MoO}_{3}$ (JCPDS 47-1320). This may be related to the phase transition because of its the long age time [21].

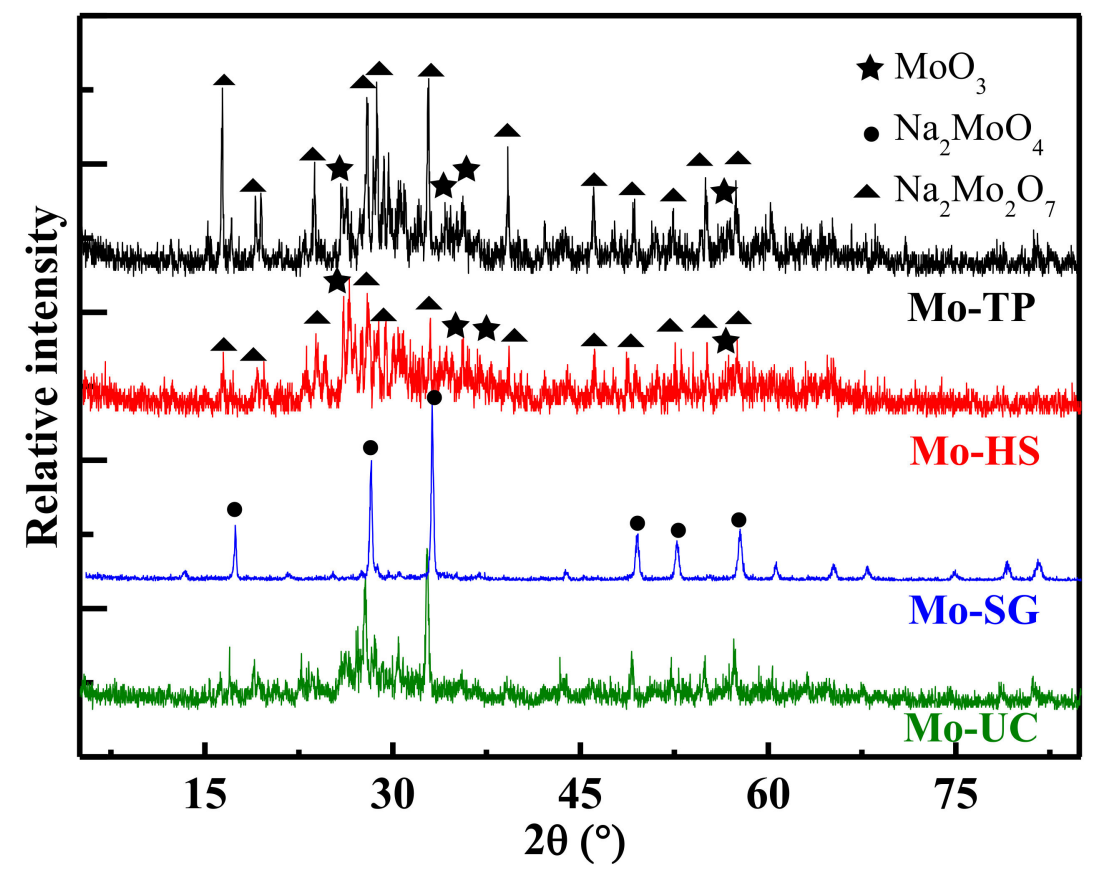

Figure 2. XRD patterns of the Mo/ZnAl-layered double hydroxide (LDH) catalysts.

\subsection{TEM Characterizations of the Mo/ZnAl-LDH Catalysts}

The morphology of Mo/ZnAl-LDH catalysts characterized by TEM are exhibited in Figure 3. The lattice lines of Mo-TP and Mo-HS, with a smaller spacing of $\sim 0.4 \mathrm{~nm}$, showed heterogeneous particle size, morphology, some groups of dark thread-like fringes, wormlike appearance, and significant order of pore arrangement, consisting of the $\mathrm{XRD}$ characterization. As shown in Figure 3C,D, Mo-SG and Mo-UC, with a smaller spacing of $\sim 0.6 \mathrm{~nm}$, structural regularization, and single crystal structure, consists of the XRD characterization. For the particle of Mo-SG, the pore structure was the exclusive morphology, while the pore diameter was significantly larger than that of Mo-TP. The plentiful appearance difference may indicate the diverse Mo species and the variance of the specific surface area. 

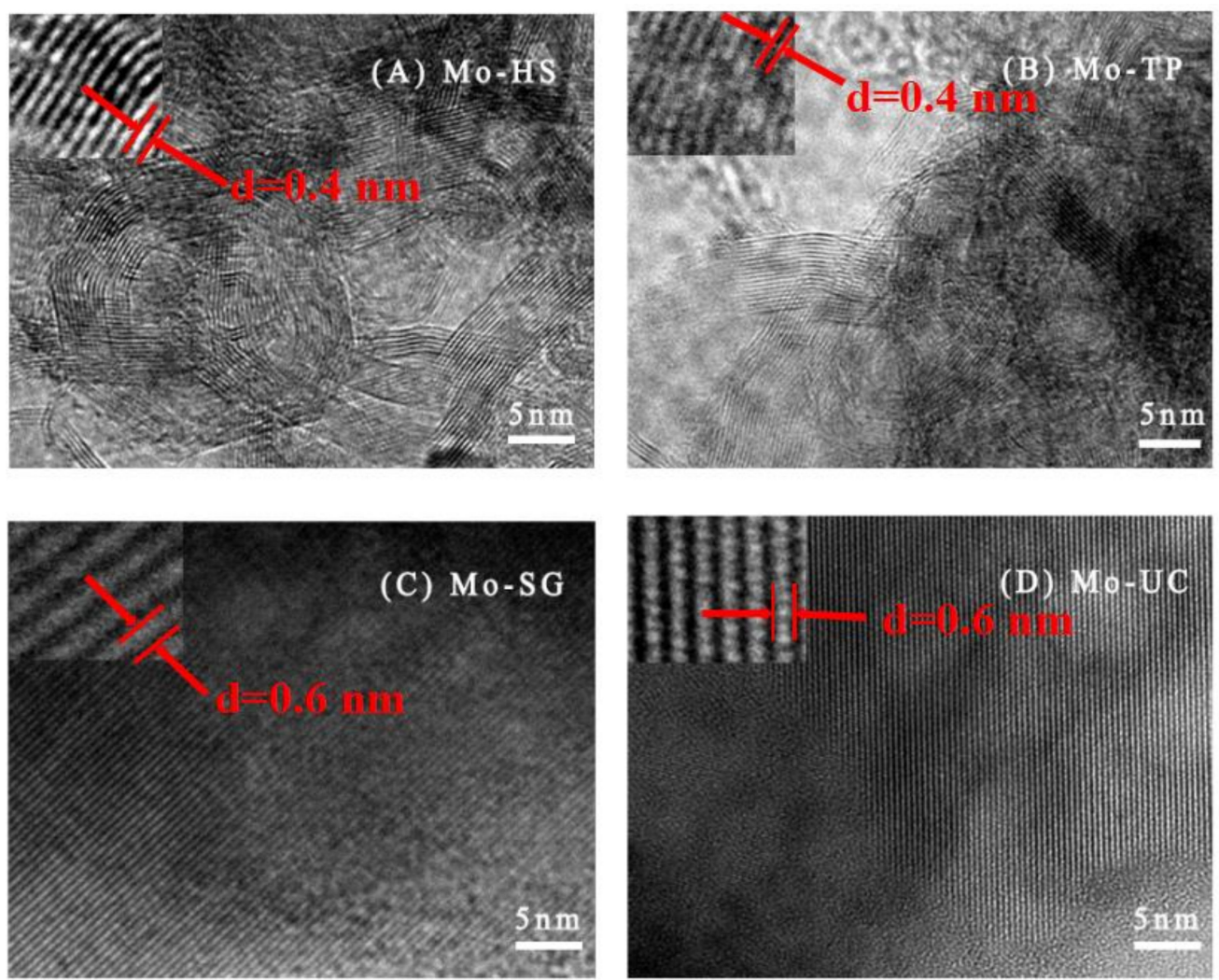

Figure 3. TEM images of Mo/ZnAl-LDH catalysts: (A) Mo-hydrothermal-synthesis (HS); (B) Mo-traditional-precipitation (TP); (C) Mo-sol-gel (SG); and (D) Mo-urea-co-precipitation (UC).

\subsection{Textural Properties of Mo/ZnAl-LDH Catalysts}

The adsorption-desorption isotherms of Mo/ZnAl-LDH catalyst are shown in Figure 4. All of the Mo/ZnAl-LDH catalysts exhibited the identical typical type IV adsorption-desorption isotherms with hysteresis loops, indicating that all catalysts showed mesoporous characteristics. BET surface area, pore size, and pore volume of all catalysts are listed in Table 1. The specific surface area of Mo-TP and Mo-HS was $2.8 \mathrm{~m}^{2} / \mathrm{g}$ and $3.9 \mathrm{~m}^{2} / \mathrm{g}$, respectively, which were almost half of the Mo-UC, which was $5.8 \mathrm{~m}^{2} / \mathrm{g}$. The specific surface area of Mo-SG was $15.4 \mathrm{~m}^{2} / \mathrm{g}$, which was nearly five times as large as that of the Mo-TP. Zeta potential was the electrical potential which could further carry the adsorption behavior of the catalysts. All catalysts showed a negative zeta potential. For example, Mo-TP and Mo-HS were $-14.4 \mathrm{mV}$ and $-18.4 \mathrm{mV}$, which were beneficial for the absorption of the cationic orchid $\mathrm{X}-\mathrm{BL}$, which is a kind of cationic azo dye with a positive Zeta potential.

Table 1. The surface area, pore diameter, and pore volume of the Mo/ZnAl-LDH catalysts.

\begin{tabular}{|c|c|c|c|c|}
\hline Catalysts & $\begin{array}{l}\text { Specific Surface Area } \\
\qquad\left(\mathrm{m}^{2} / \mathrm{g}\right)\end{array}$ & $\begin{array}{l}\text { Pore Volume } \\
\qquad\left(\mathrm{cm}^{3} / \mathrm{g}\right)\end{array}$ & $\begin{array}{l}\text { Pore Diameter } \\
(\mathrm{nm})\end{array}$ & $\begin{array}{l}\text { Zeta Potential } \\
(\mathrm{mV})\end{array}$ \\
\hline Mo-TP & 2.8 & 0.03 & 3.8 & -14.4 \\
\hline Mo-HS & 3.9 & 0.01 & 3.8 & -18.4 \\
\hline Mo-SG & 15.4 & 0.07 & 12.6 & -20.2 \\
\hline Mo-UC & 5.8 & 0.04 & 2.2 & -11.2 \\
\hline
\end{tabular}




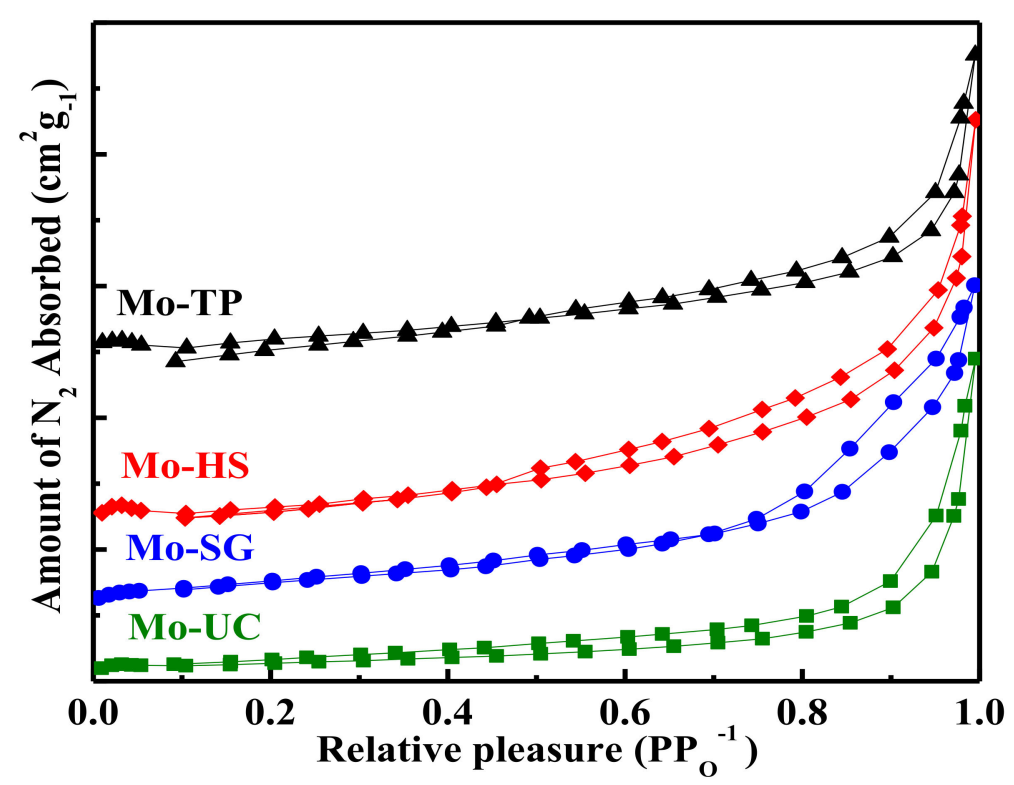

Figure 4. $\mathrm{N}_{2}$ adsorption-desorption isotherm curves of the Mo/ZnAl-LDH catalysts.

\subsection{XPS Characterization of the Mo/ZnAl-LDH Catalysts}

XPS is an efficient method to investigate the surface composition and chemical state of the elements on the surface of as-prepared catalysts. Figure 5 shows the full XPS survey spectra of the Mo/ZnAl-LDH catalysts. Chemical composition of the Mo/ZnAl-LDH catalysts, with supports prepared using different methods, are shown in Table 2. The peaks of $\mathrm{Mo}, \mathrm{Zn}, \mathrm{Al}$, and $\mathrm{O}$ were observed in all as-prepared catalysts. The element of zinc in the Mo-SG showed little change, as Zn 2p core-level spectra was almost completely transformed into the Zn-LMM. The percentage of Mo dosing in the Mo-TP and Mo-HS, were higher than that of the Mo-SG and Mo-UC, which meant that the TP and HS method promoted interactions between the Mo and the LDHs.

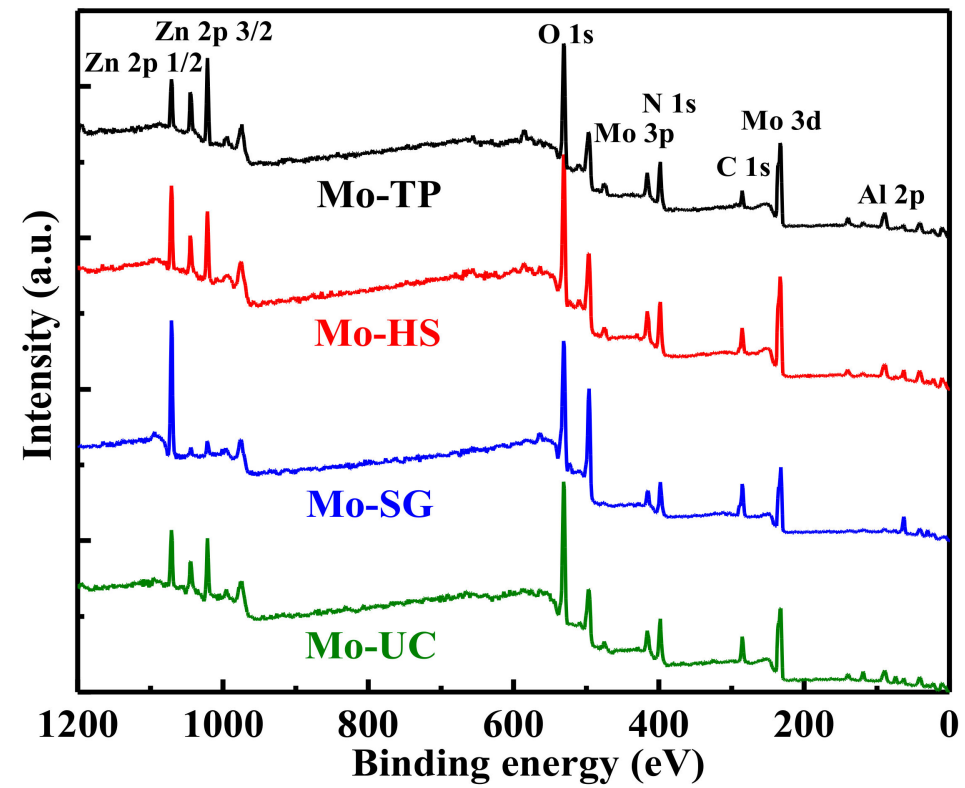

Figure 5. The X-ray photoelectron spectroscopy (XPS) survey spectra of the Mo/ZnAl-LDH catalysts. 
Table 2. Chemical composition obtained by the XPS spectra of the Mo/ZnAl-LDH catalysts.

\begin{tabular}{ccccccc}
\hline Catalysts & Mo (at \%) & Zn (at \%) & Al (at \%) & O (at \%) & Na (at \%) & C (at \%) \\
\hline Mo-TP & 12.7 & 2.4 & 1.1 & 48.6 & 8.0 & 27.1 \\
Mo-HS & 15.0 & 3.5 & 0.7 & 55.8 & 7.4 & 17.7 \\
Mo-SG & 8.3 & 0.3 & 1.5 & 44.3 & 12.8 & 32.8 \\
Mo-UC & 10.3 & 2.0 & 6.7 & 23.4 & 5.4 & 52.2 \\
\hline
\end{tabular}

All Mo 3d XPS spectra exhibited the characteristic 3d 5/2 and 3d 3/2 doublet, caused by spin-orbit coupling of the Mo $3 \mathrm{~d}$ orbitals (Figure 6). For the Mo-SG and the Mo-UC, a single assumption of a relative intensity ratio of the Mo $3 \mathrm{~d} 5 / 2$ and Mo $3 \mathrm{~d} 3 / 2$ lines, at $232.2 \mathrm{eV}$ and $235.4 \mathrm{eV}$, was fixed to 3:2 which revealed two peaks for each Mo 3d 5/2 and Mo 3d 3/2 spin-orbit coupling [22,23]. For the Mo-TP and Mo-HS, the characteristic binding energy separation ( $\Delta$ Mo $3 d$ ) between the Mo $3 \mathrm{~d} 5 / 2$ and $3 \mathrm{~d} 3 / 2$ was $\sim 3.1 \mathrm{eV}$, as observed previously in the literature [24]. The tiny chemical shift of the Mo $3 \mathrm{~d}$ $5 / 2$ at $231.8 \mathrm{eV}$ and $\mathrm{Mo} 3 \mathrm{~d} 3 / 2$ at $234.9 \mathrm{eV}$ implied that a tiny difference of the chemical composition and content occurred in the Mo/ZnAl-LDH catalysts. The Mo-TP and Mo-HS had a lower energy binding of Mo that was easier to transform into other valence states.

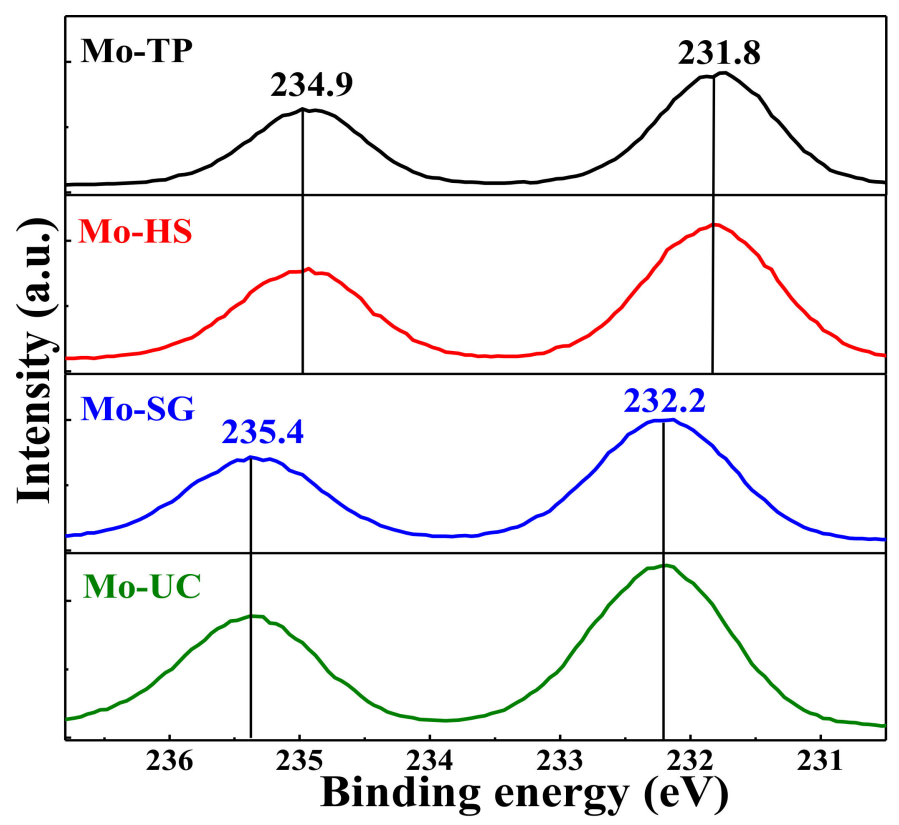

Figure 6. Mo 3d 3/2 and Mo 3d 5/2 peaks of the Mo/ZnAl-LDH catalysts.

More information can be obtained from the comparison of the O 1s XPS spectra (Figure 7). Notably, both adsorption oxygen with a high binding energy and lattice oxygen with low binding energy, existed on the surface of the catalysts. The peak $\mathrm{O} 1 \mathrm{~s}$ of the $529-531 \mathrm{eV}$ corresponded to the $\mathrm{O}^{2-}$ ions, in the metal oxide, and the binding energy in the region of 531-533 eV was associated with surface adsorbed oxygen, from either surface hydroxyl or carbonate species. The peak after $532 \mathrm{eV}(\mathrm{BE})$ was associated with adsorbed molecular water. The evidence was that the amount of $\mathrm{O}^{2-}$ ions in the Mo-TP and the Mo-HS was larger than in the Mo-SG and Mo-UC. The presence of surface hydroxyl or carbonate species played an important role in the selective oxidation process. However, large surface adsorbed oxygen could inhibit the catalytic activity. 

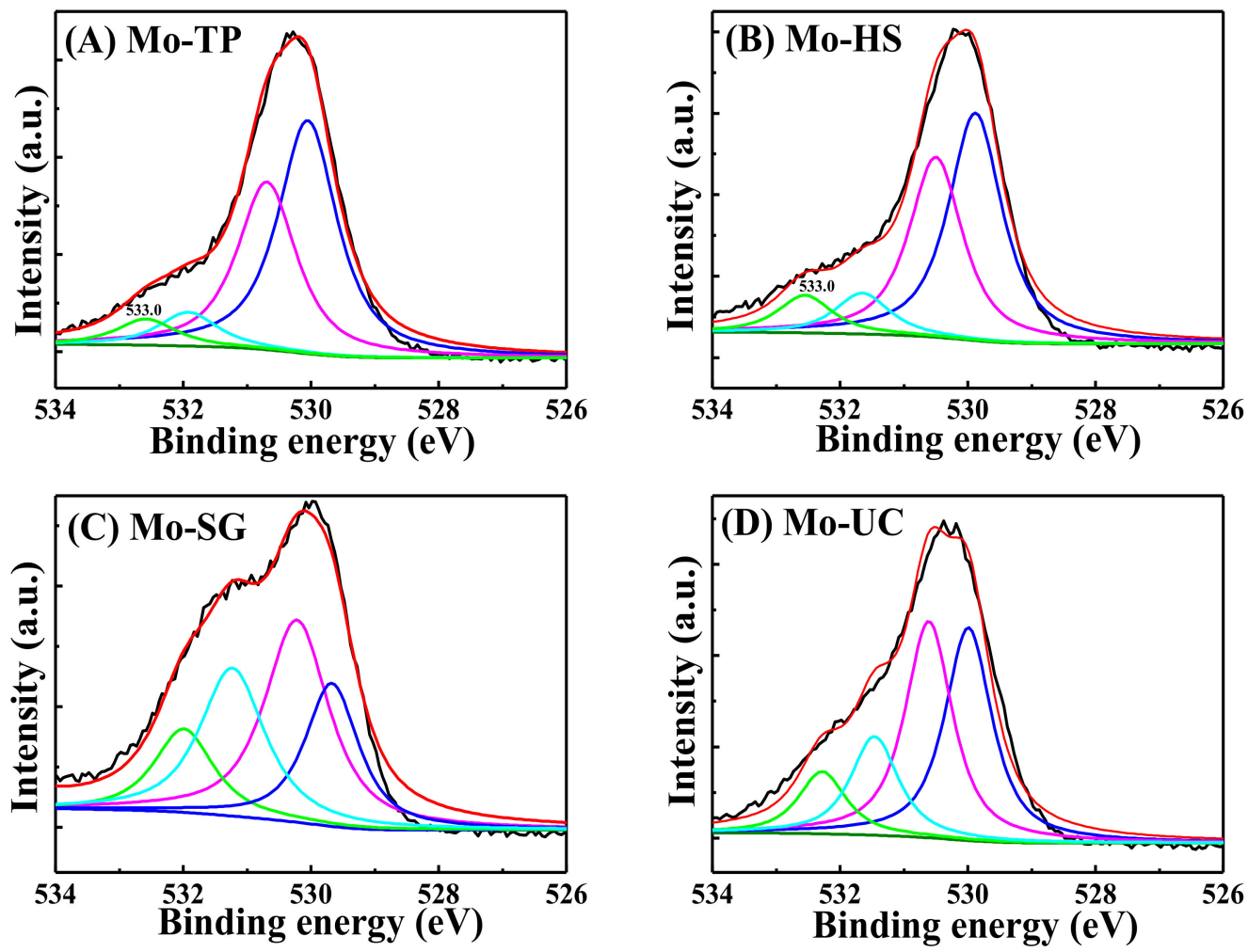

Figure 7. O 1s for the Mo/ZnAl-LDH catalysts: (A) Mo-traditional-precipitation (TP); (B) Mo-hydrothermalsynthesis (HS); (C) Mo-sol-gel (SG); and (D) Mo-urea-co-precipitation (UC).

\subsection{CWAO Activity of the Mo/ZnAl-LDH Catalysts}

The decolorization of the cationic orchid X-BL by the Mo/ZnAl-LDH catalysts was investigated in the CWAO process, under room temperature and pressure. In addition, mineralization efficiency of the cationic orchid X-BL was examined by the TOC analysis. As illustrated in Figure 8a,b, Mo-TP and Mo-HS showed better catalytic performance, compared with Mo-SG and Mo-UC. The superior decolorization ratio and the TOC removal efficiency of the cationic orchid X-BL were $84.2 \%$ and $73.9 \%$, by Mo-TP, and $84.8 \%$ and $71.0 \%$, by Mo-HS, respectively. To further verify the catalytic activity of the Mo/ZnAl-LDH catalysts, the adsorption efficiency was also measured for each sample (Figure 8c). The adsorption efficiency of all nano-hybrids was below $20 \%$, suggesting that a catalytic reaction took place in the presence of air in the CWAO process. In general, the specific surface area was positive contact to the catalytic activity, as a catalyst with larger specific surface area could acquire more access with pollutants. Interestingly, the Mo-TP and Mo-HS catalysts possessed excellent catalytic activities with smaller specific surface area, as compared to the Mo-SG and Mo-UC. It was reasonable that $\mathrm{Na}_{2} \mathrm{Mo}_{2} \mathrm{O}_{7}$ was formed in the preparation of the Mo-TP and the Mo-HS. $\mathrm{Na}_{2} \mathrm{Mo}_{2} \mathrm{O}_{7}$ possessed an excellent catalytic activity and $\mathrm{Na}_{2} \mathrm{MoO}_{4}$ was not the active species, which was quite in line with the result [25-27]. In addition, $\mathrm{O}^{2-}$ ions in the oxygen deficient regions in Mo-TP and Mo-HS promoted the formation of active $\cdot \mathrm{OH}$ radicals [28]. The highest catalytic performance could be credited to appropriate crystalline from the XRD analysis, specific Mo species, lower zeta potential, higher active component of Mo and unique microgranular texture. Recycling was a parameter for evaluating the stability of the catalyst [29], and its results are show in Figure 8d. The discoloration rate by the Mo-TP and Mo-HS remained above 60\%, after three reactions, however, the Mo-SG and Mo-UC were just under $20 \%$ of the discoloration rate, after two reactions. Therefore, the improvement of chemical stability for the Mo/ZnAl-LDH catalyst will be studied further in the next work. 

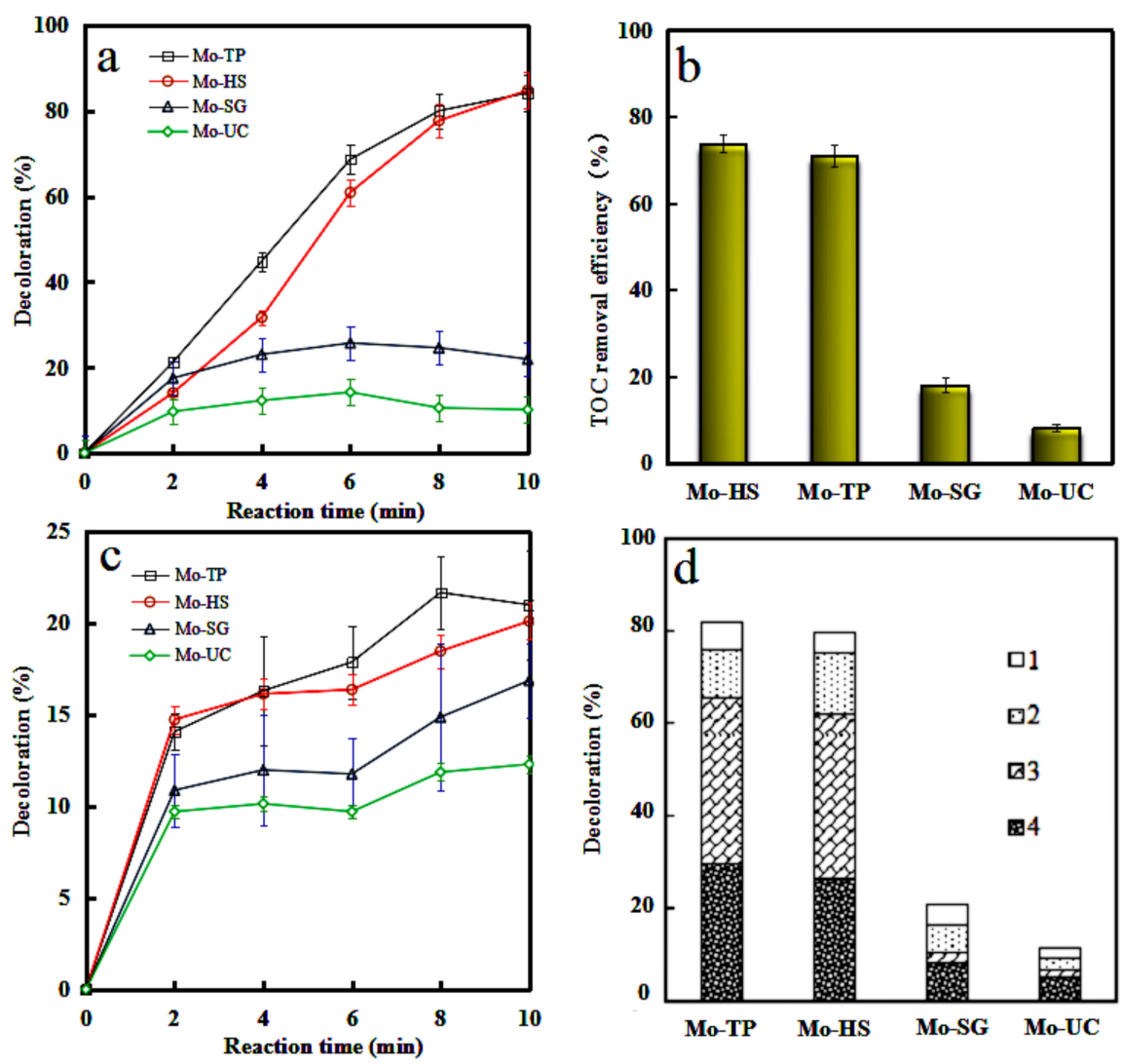

Figure 8. The degradation of Cationic Orchid X-BL $(\mathbf{a}, \mathbf{b})$ by the Mo/ZnAl-LDH catalysts in catalytic wet air oxidation (CWAO); the adsorption of Cationic Orchid X-BL (c) by the Mo/ZnAl-LDH catalysts; the recycling times $(\mathbf{d})$ of the Mo/ZnAl-LDH catalysts.

\section{Conclusion}

The carrier of $\mathrm{Zn}-\mathrm{Al} \mathrm{LDH}$ with the four preparation methods have an influence on the catalytic performance of the Mo/ZnAl-LDH catalysts. The Mo-TP catalyst and the Mo-HS catalyst showed high catalytic performance for the degradation of cationic orchid X-BL, under room temperature and pressure. $\mathrm{Na}_{2} \mathrm{Mo}_{2} \mathrm{O}_{7}$ was detected in the Mo-TP and the Mo-HS, which contributed to the notably high catalytic activity in the organic wastewater degradation. The excellent performance could be attributed to the specific Mo species, low zeta position, high degree of Mo dispersion, and the unique texture properties.

Author Contributions: Y.L. (Yang Li) and T.L. conceived and designed the experiments; Y.L. (Yang Li) and T.L. performed the experiments and analyzed the data; Y.X. contributed reagents/materials/analysis tools; Y.L. (Yun Liu) and F.G. supervised the research; Y.X. and T.L. revised the manuscript.

Funding: This research is supported by the National Natural Science Foundation of China (NSFC: 51308484 and 51678511), and Major Talent Training Program of the Xiangtan University (16PYZ09).

Acknowledgments: We gratefully acknowledge the financial support from the National Natural Science Foundation of China (51308484 and 51678511), Major Talent Training Program of the Xiangtan University (16PYZ09).

Conflicts of Interest: The authors declare no conflict of interest.

\section{References}

1. Rodriguez, A.; Garcia, J.; Ovejero, G.; Mestanza, M. Wet air and catalytic wet air oxidation of several azodyes from wastewaters: the beneficial role of catalysis. Water Sci. Technol. 2009, 60, 1989-1999. [CrossRef] [PubMed]

2. Qiu, G.; Song, Y.; Zeng, P.; Xiao, S.; Duan, L. Phosphorus recovery from fosfomycin pharmaceutical wastewater by wet air oxidation and phosphate crystallization. Chemosphere 2011, 84, 241-246. [CrossRef] [PubMed] 
3. Xu, Y.; Shao, H.; Ge, F.; Liu, Y. Novel-structured Mo-Cu-Fe-O composite for catalytic air oxidation of dye-containing wastewater under ambient temperature and pressure. Chinese J. Catal. 2017, 38, 1719-1725. [CrossRef]

4. Liu, Y.; Sun, D. Development of $\mathrm{Fe}_{2} \mathrm{O}_{3}-\mathrm{CeO}_{2}-\mathrm{TiO}_{2} / \gamma-\mathrm{Al}_{2} \mathrm{O}_{3}$ as catalyst for catalytic wet air oxidation of methyl orange azo dye under room condition. Appl. Catal. B Environ. 2007, 72, 205-211. [CrossRef]

5. Vallet, A.; Ovejero, G.; Rodríguez, A.; Peres, J.A.; García, J. Ni/MgAlO regeneration for catalytic wet air oxidation of an azo-dye in trickle-bed reaction. J. Hazard. Mater. 2013, 244, 46-53. [CrossRef] [PubMed]

6. Liu, J.; Yu, C.; Zhao, P.; Chen, G. Comparative study of supported $\mathrm{CuO}_{\mathrm{x}}$ and $\mathrm{MnO}_{\mathrm{x}}$ catalysts for the catalytic wet air oxidation of $\beta$-naphthol. Appl. Surf. Sci. 2012, 258, 9096-9102. [CrossRef]

7. Xu, Y.; Li, X.; Cheng, X.; Sun, D.; Wang, X. Degradation of cationic red GTL by catalytic wet air oxidation over Mo-Zn-Al-O catalyst under room temperature and atmospheric pressure. Environ. Sci. Technol. 2012, 46, 2856-2863. [CrossRef] [PubMed]

8. Xu, Y.; Chen, X.Y.; Li, Y.; Ge, F.; Zhu, R.L. Quantitative structure-property relationship (QSPR) study for the degradation of dye wastewater by Mo-Zn-Al-O catalyst. J. Mol. Liq. 2016, 215, 461-466. [CrossRef]

9. Wu, J.; Xia, Q.; Wang, H.; Li, Z. Catalytic performance of plasma catalysis system with nickel oxide catalysts on different supports for toluene removal: Effect of water vapor. Appl. Catal. B Environ. 2014, 156, 265-272. [CrossRef]

10. Gutiérrez, O.Y.; Klimova, T. Effect of the support on the high activity of the (Ni) $\mathrm{Mo} / \mathrm{ZrO}_{2}-\mathrm{SBA}-15$ catalyst in the simultaneous hydrodesulfurization of DBT and 4, 6-DMDBT. J. Catal. 2011, 281, 50-62. [CrossRef]

11. Yoosuk, B.; Song, C.; Kim, J.H.; Ngamcharussrivichai, C.; Prasassarakich, P. Effects of preparation conditions in hydrothermal synthesis of highly active unsupported NiMo sulfide catalysts for simultaneous hydrodesulfurization of dibenzothiophene and 4, 6-dimethyldibenzothiophene. Catal. Today 2010, 149, $52-61$. [CrossRef]

12. Infantes-Molina, A.; Moreno-León, C.; Pawelec, B.; Fierro, J.; Rodríguez-Castellón, E.; Jiménez-López, A. Simultaneous hydrodesulfurization and hydrodenitrogenation on $\mathrm{MoP} / \mathrm{SiO}_{2}$ catalysts: Effect of catalyst preparation method. Appl. Catal. B Environ. 2012, 113, 87-99. [CrossRef]

13. Zhou, Q.; Zhu, R.; Parker, S.C.; Zhu, J.; He, H.; Molinari, M. Modelling the effects of surfactant loading level on the sorption of organic contaminants on organoclays. RSC Adv. 2015, 5, 47022-47030. [CrossRef]

14. Zgolicz, P.D.; Stassi, J.P.; Yañez, M.J.; Scelza, O.A.; de Miguel, S.R. Influence of the support and the preparation methods on the performance in citral hydrogenation of Pt-based catalysts supported on carbon nanotubes. J. Catal. 2012, 290, 37-54. [CrossRef]

15. Li, X.; Zou, X.; Qu, Z.; Zhao, Q.; Wang, L. Photocatalytic degradation of gaseous toluene over Ag-doping $\mathrm{TiO}_{2}$ nanotube powder prepared by anodization coupled with impregnation method. Chemosphere 2011, 83, 674-679. [CrossRef] [PubMed]

16. Skaf, M.; Aouad, S.; Hany, S.; Cousin, R.; Abi-Aad, E.; Aboukaïs, A. Physicochemical characterization and catalytic performance of $10 \% \mathrm{Ag} / \mathrm{CeO}_{2}$ catalysts prepared by impregnation and deposition-precipitation. J. Catal. 2014, 320, 137-146. [CrossRef]

17. Qian, K.; Fang, J.; Huang, W.; He, B.; Jiang, Z.; Ma, Y.; Wei, S. Understanding the deposition-precipitation process for the preparation of supported Au catalysts. J. Mol. Catal. A Chem. 2010, 320, 97-105. [CrossRef]

18. Chubar, N.; Gerda, V.; Megantari, O.; Mičušík, M.; Omastova, M.; Heister, K.; Man, P.; Fraissard, J. Applications versus properties of $\mathrm{Mg}$-Al layered double hydroxides provided by their syntheses methods: Alkoxide and alkoxide-free sol-gel syntheses and hydrothermal precipitation. Chem. Eng. J. 2013, 234, 284-299. [CrossRef]

19. Wang, D.; Pan, Z.; Wu, Z.; Wang, Z.; Liu, Z. Hydrothermal synthesis of $\mathrm{MoS}_{2}$ nanoflowers as highly efficient hydrogen evolution reaction catalysts. J. Power Sources 2014, 264, 229-234. [CrossRef]

20. Li, Y.; Xu, Y.; Chen, X.Y.; Ge, F.; Zhu, R.L. High catalytic activity of Mo-Zn-Al-O catalyst for dye degradation: Effect of $\mathrm{pH}$ in the impregnation process. Appl. Catal. B Environ. 2014, 160, 115-121. [CrossRef]

21. Xu, Y.; Sun, D. Structure and catalytic activity of Mo-Zn-Al-O catalyst for degradation of cationic red GTL under room conditions. Chem. Eng. J. 2012, 183, 332-338. [CrossRef]

22. Mudher, K.S.; Keskar, M.; Krishnan, K.; Venugopal, V. Thermal and X-ray diffraction studies on $\mathrm{Na}_{2} \mathrm{MoO}_{4}$, $\mathrm{Na}_{2} \mathrm{Mo}_{2} \mathrm{O}_{7}$ and $\mathrm{Na}_{2} \mathrm{Mo}_{4} \mathrm{O}_{13}$. J. Alloy Compd. 2005, 396, 275-279. [CrossRef] 
23. Zimowska, M.; Łątka, K.; Mucha, D.; Gurgul, J.; Matachowski, L. The continuous conversion of ethanol and water mixtures into hydrogen over $\mathrm{Fe}_{\mathrm{x}} \mathrm{O}_{\mathrm{y}} / \mathrm{MoO}_{3}$ catalytic system-XPS and Mössbauer studies. J. Mol. Catal. A Chem. 2016, 423, 92-104. [CrossRef]

24. Quincy, R.B.; Houalla, M.; Proctor, A.; Hercules, D.M. Distribution of molybdenum oxidation states in reduced molybdenum/titania catalysts: correlation with benzene hydrogenation activity. J. Phys. Chem. 1990, 94, 1520-1526. [CrossRef]

25. Belanger, D.; Laperriere, G. Electrochromic molybdenum trioxide thin film preparation and characterization. Chem. Mater. 1990, 2, 484-486. [CrossRef]

26. Zhang, Y.; Zhang, Z.; Zhou, T.; Lu, P.; Gao, Y.; Yu, F.; Umar, A.; Wang, Q. Synthesis and characterization of alkali metal molybdates with high catalytic activity for dye degradation. RSC Adv. 2016, 6, 54553-54563. [CrossRef]

27. Yang, R.; Zhang, Z.; Umar, A.; Gao, Y.; Wang, J.; Lu, P.; Guo, Z.; Huang, L.; Zhou, T.; Wang, Q. Preparation of $\mathrm{Ni}$ and Fe doped molybdate-based catalyst from Ni-Fe layered double hydroxide for the catalytic wet air oxidation of dyes. Sci. Adv. Mater. 2015, 7, 1435-1442. [CrossRef]

28. Zhang, Z.; Yang, R.; Gao, Y.; Zhao, Y.; Wang, J.; Huang, L.; Guo, J.; Zhou, T.; Lu, P.; Guo, Z. Novel $\mathrm{Na}_{2} \mathrm{Mo}_{4} \mathrm{O}_{13} / \alpha-\mathrm{MoO}_{3}$ hybrid material as highly efficient CWAO catalyst for dye degradation at ambient conditions. Sci. Rep. 2014, 4, 6797. [CrossRef] [PubMed]

29. Shokouhimehr, M.; Hong, K.; Lee, T.H.; Moon, C.W.; Hong, S.P.; Zhang, K.; Suh, J.M.; Choi, K.S.; Varma, R.S.; Jang, H.W. Magnetically retrievable nanocomposite adorned with Pd nanocatalysts: Efficient reduction of nitroaromatics in aqueous media. Green Chem. 2018, 20, 3809-3817. [CrossRef]

(C) 2018 by the authors. Licensee MDPI, Basel, Switzerland. This article is an open access article distributed under the terms and conditions of the Creative Commons Attribution (CC BY) license (http:/ / creativecommons.org/licenses/by/4.0/). 